\title{
Risk Factors for Neonatal Sepsis and Method for Reduction of Blood Culture Contamination
}

\section{S S -Krajčinović ${ }^{1}$, A Doronjski ${ }^{1,2}$, N Barišićc ${ }^{1,2}$, V Stojanović ${ }^{1,2}$}

1.Intensive Care Unit, Institute for Child and Youth Health care of Vojvodina, Hajduk Velikova 10, 21000 Novi Sad, Serbia

2. Medical faculty, University of Novi Sad, Hajduk Veljkova 3, 21000 Novi Sad, Serbia

Correspondence to: Nenad Barišić,

Email: barisicnenadns@gmail.com

\begin{abstract}
Background

False-positive blood cultures findings may lead to a falsely increased morbidity and increased hospital costs.

Method

The survey was conducted as retrospective - prospective study and included 239 preterm infants (born before 37 weeks of gestation) who were treated in Neonatal Intensive Care Unit (NICU) in Institute for Child and Youth Health Care of Vojvodina during one year (January 1st, 2012 to December 31st, 2012). The retrospective part of the study focused on examination of incidence of neonatal sepsis and determination of risk factors. In the prospective part of the study infants were sub-divided into two groups: Group 1-infants hospitalized in NICU during the first 6 months of the study; blood cultures were taken by the "clean technique" and checklists for this procedure were not taken. Group 2- neonates hospitalized in NICU during last 6 months of the study; blood cultures were taken by "sterile technique" and checklists for this procedure were taken.
\end{abstract}

\section{Results}

The main risk factors for sepsis were prelabor rupture of membranes, low gestational age, low birth weight, mechanical ventilation, umbilical venous catheter placement, and abdominal drainage. Staphylococcus aureus and coagulase negative Staphylococcus were the most frequently isolated microorganisms in false-positive blood samples.

\section{Conclusions}

Education of employees, use of checklists and sterile sets for blood sampling, permanent control of false positive blood cultures, as well as regular and routine monthly reports are crucial for successful reduction of contamination rates.

\section{Introduction}

Neonatal sepsis is an invasive bacterial infection that occurs in newborns between first and ninetieth day of life. Neonatal sepsis is one of the most frequent causes of morbidity and mortality in newborns ${ }^{1}$. Worldwide, incidence of sepsis in newborns varies between 1- 10 cases per 1000 live births, and the mortality varies between $15-50 \%{ }^{2}$. Neonatal sepsis is usually classified as early or late sepsis. Early sepsis occurs within the first 72 hours of birth and late neonatal sepsis occurs after 72 hours of birth. Early neonatal sepsis is associated with acquisition of microorganism from mother (transplacental infection, ascending infection from cervix and the newborn can acquire the microorganisms as it passes through the birth canal at delivery). Late neonatal sepsis usually occurs due to the lack of aseptic working conditions during the placement and maintenance of intravenous cannulas, central venous catheters, umbilical catheters, thoracic and lumbar puncture, application of parenteral therapy and infusion ${ }^{3-5}$. Having in mind that nurses participate in these medico-technical procedures, either individually or as a member of the multidisciplinary team, the occurrence of late sepsis is considered an important indicator of quality of care. Risk factors for sepsis in premature infants have been the subject of many studies. Most of them came to the conclusion that commonest risk factors are preterm birth, small gestational age, low birth weight, twin pregnancy and congenital anomalies, ${ }^{6,7}$. Gold standard for isolation and verification of microbial pathogens of sepsis are blood cultures. Positive blood cultures findings, in a setting where such findings do not fit in the general condition of the child and where his/hers laboratory tests (i.e. C-reactive protein) are negative, are common in every day practice. False-positive blood cultures are usually consequence of contamination of the blood samples by the bacteria from the skin and the environment of the child. False-positive blood cultures are a big problem in practice, because such findings often lead to a falsely high morbidity and higher hospital costs ${ }^{8}$. Premature infants with false-positive blood cultures usually are treated as children with sepsis ${ }^{3}$. The aim of this study was to determine the incidence of sepsis in premature infants treated in the intensive care unit as well as to determine the risk factors and aetiology of sepsis, and to determine if preventive medicotechnical measures (sterile technique of sampling for blood culture and checklists) lead to significant reduction in falsepositive blood cultures.

\section{Methods}

The survey was conducted as retrospective - prospective study and included 239 preterm infants (born before 37 weeks of gestation) who were treated in Neonatal Intensive Care Unit (NICU) in Institute for Child and Youth Health Care of Vojvodina during one year (January 1st, 2012 to December 31st, 2012). The local institutional review board approved this study. The first (retrospective) part of the study focused on the examination of the incidence of neonatal sepsis and determination of risk factors. Data were analyzed from the medical records of all prematurely born newborns addmited in NICU from January 1st to June 31st 2012. During this period, blood specimens for blood cultures were taken by the "clean technique" and checklists for this procedure were not taken.These children were designated in Group 1. In all subjects, we analyzed several variables:

The risk factors from the preterm infants: gestational age, weight, sex, Apgar score in the first and fifth minute, the method of delivery, intrauterine growth restriction, congenital anomalies, amniotic fluid (appearance, odour, quantity), mechanical ventilation, resuscitation at birth, necrotizing enterocolitis pneumonia, meningitis, ventriculoperitoneal shunts, and presence of abdominal drainage.

- Maternal risk factors: maternal infections, hypertension, premature rupture of membranes, abortions, multiple pregnancies, artificial insemination. The second part of the study (prospective part), included all prematurely born neonates who were hospitalized in NICU from July 1st to December 31st 2012. During this period blood cultures were taken by "sterile technique" and checklists for this procedure were taken. Children involved in the study during this period were designated in Group 2. All specimens for microbiological analysis were collected by NICU nurses. 
In group 1 no standarized technique for blood culture collection was used (nurses used nonsterile gloves, no well-defined sterile field, 70\% isopropyl alcohol and $0.1 \%$ butanediol for skin antisepsis). Checklists for the procedure were not provided. That was "clean technique". In group 2, a sterile sampling technique was used for blood specimen collection (sterile gloves, sterile field, sterile gauzes, $70 \%$ isopropyl alcohol and $0.1 \%$ butanediol for skin antisepsis). Sterile kits were not made. Checklists for the procedure were provided. Before the introduction of sterile technique, nursing educators trained the NICU staff how to conduct the procedure. Blood specimens were inoculated in Bac'T/ ALERT® Culture Media (Biomerieux, INC, Durham) and sent to analysis to local reference laboratory. In all patients who had positive blood culture findings, additional data were taken: the type of bacteria, and the origin of the sample (peripheral vein, umbilical venous catheter). In those cases, confirmation of the diagnosis of neonatal sepsis was based on positive clinical findings (food intolerance, vomiting, diarhoea or abdominal distension, tachypnea, respiratory distress, apnoea, tachycardia, hypotension, hyper- or hypoglycaemia, metabolic acidosis, hypo- or hyperthermia, etc.) and laboratory findings (elevated levels of serum $\mathrm{C}$ reactive protein and/or procalcitonin, leucocytosis/ leucopoenia, thrombocytopenia, etc.). If those features were absent and if before blood sampling patient did not receive antibiotics, positive findings of blood cultures were considered as contamination. For descriptive statistics absolute numbers, percentages and measures of central tendency (mean, median, standard deviation, minimum and maximum values) were used. For statistical testing, $\chi{ }^{2}$ test and t-test were used as appropriate. In the applied tests, the level of significance were set at 95\% ( $<<0.05)$.

\section{Results}

The study included 239 preterm infants, of whom $27 \%$ ( $\mathrm{n}=63 / 239)$ developed neonatal sepsis, and 73\% ( $\mathrm{n}=$ $176 / 239)$ did not. The group 1 included 56\% $(\mathrm{n}=134 / 239)$, a group $2,44 \%(n=105 / 239)$ of preterm infants. Of the total number of subjects who were diagnosed with sepsis, $48 \%(\mathrm{n}=30 / 63)$ had an early sepsis, and $52 \%(\mathrm{n}=33 / 63)$ late sepsis. The incidence of early and late sepsis among neonates in group 1 did not differ significantly from the incidence of early and late sepsis among neonates in group 2 (early sepsis: $\mathrm{p}=0.592$ and late sepsis: $\mathrm{p}=0.592$ ). Table 1 shows characteristics of the newborns and comorbidity in newborns with and without sepsis. Infants who had sepsis were significantly more likely to be on mechanical ventilation and more succeptable for comorbidities (pneumonia, necrotizing enterocolitis, meningitis) and more frequently had implants such as ventriculoperitoneal shunt, abdominal drains or umbilical venous catheters $(p<0.05)$. Infants with sepsis had significantly lower birth weight(1325g vs $1749 \mathrm{~g}$, $\mathrm{p}<0.05$ ) and lower gestational age ( 29 vs. 31 weeks, $\mathrm{p}<0.05)$ than infants without sepsis. Negative outcome (death) was more common in the group of prematurely born infants who had sepsis than in those who did not have sepsis $(33.3$ $\%$ vs $8.5 \%, \mathrm{p}<0.05)$. Table 2 shows that premature rupture of membranes was strongly associated with the onset of sepsis $(22.2 \%$ vs $9.7 \%, \mathrm{p}<0.05)$.

Table 3 shows the distribution of Apgar scores in the first and fifth minute of life. Newborns with sepsis were more likely to have Apgar score in the first minute $\leq 4$ (49.2\% vs 28.9 $\%, \mathrm{p}<0.05)$, and Apgar score in the fifth minute 5-7 $(55.6 \%$ vs $35.8 \%, \mathrm{p}<0.05)$ in comparation to infants without sepsis . Acinetobacter spp. was the most frequently isolated bacterial species in blood cultures of newborns with confirmed sepsis. Also, Acinetobacter spp. is the most common cause of early and late neonatal sepsis (Table 4) Table 5 shows the most common causes of false-positive blood cultures in group 1 and group 2. Staphylococcus aureus $(p=0.006)$ and Staphylococcus spp - coagulase negative

$(p=0.07)$ were the most frequently isolated microorganisms in false-positive blood samples in group 1 and group 2, respectivelly. Of the total number of blood cultures taken in group 1, contaminated blood cultures accounts for $16.4 \%$ ( $\mathrm{n}=22 / 134)$, and in group 2, contaminated blood cultures accounts for $7.6 \%(n=8 / 105)(p=0.025)$.

Table 1. Characteristics of the newborns and co morbidity in newborns with and without sepsis.

\begin{tabular}{|c|c|c|c|}
\hline & $\begin{array}{l}\text { Sepsis } \\
(n-63)\end{array}$ & $\begin{array}{l}\text { Without sepsis } \\
\qquad(n-176)\end{array}$ & $\mathbf{p}$ \\
\hline Gestational age (weeks) & $29(24-36)$ & $31(23-36.5)$ & $p<0.05$ \\
\hline Birth weight (g) & $1325(600-3400)$ & $1749(510-3600)$ & $p<0.05$ \\
\hline Male/Female & $36 / 27(57.1 \% / 42.9 \%)$ & $93 / 83(52.8 \% / 47.2 \%)$ & $p>0.05$ \\
\hline Birth delivery mode & & & $p>0.05$ \\
\hline -vaginal & $44.4 \%(28 / 63)$ & $57.9 \%(102 / 176)$ & \\
\hline -Caesarean Section & $55.6 \%(35 / 63)$ & $42.1 \%(74 / 1776)$ & \\
\hline Congenital malformations & $6 \%(4 / 63)$ & $(3.9 \%) 7 / 176$ & $p \rightleftharpoons 0.05$ \\
\hline Intrauterine growth restriction & $22.2 \%(14 / 63)$ & $21 \%(37 / 176)$ & $p>0.05$ \\
\hline Mechanical ventilation & $82.5 \%(52 / 63)$ & $65.9 \%(116 / 176)$ & $\mathrm{p}<0,05$ \\
\hline Noninvasive ventilation & $4.8 \%(3.63)$ & $17 \%(30 / 176)$ & $p<0.05$ \\
\hline Umbilical venous catheter & $41,3 \%(26,63)$ & $6,9 \%(12 / 176)$ & $p<0.05$ \\
\hline P'neumonia & $38.1 \%(24 / 63)$ & $12,5 \%(22 / 176)$ & $p<0.05$ \\
\hline Necrotizing enterocolitis & $25.4 \%(16 / 63)$ & $5,1 \%(9 / 176)$ & $p<0.05$ \\
\hline Meningitis & $7,9 \%(5 / 63)$ & $0,6 \%(1 / 176)$ & $p<0.05$ \\
\hline VP shunt & $3,2 \%(2 / 63)$ & $0.6 \%(1 / 176)$ & $p \curvearrowright 0.05$ \\
\hline Abdominal drain & $9.5 \%(6 / 63)$ & $1.7 \%(3 / 176)$ & $p<0.05$ \\
\hline Death & $33.3 \%(21 / 63)$ & $8.5 \%(15 / 176)$ & $p<0.05$ \\
\hline
\end{tabular}

Table 2. Complications during pregnancy in the groups of newborns with and without sepsis.

\begin{tabular}{|c|c|c|c|}
\hline & Sepsis & Without sepsis & \\
\hline Complicutions during pregnancy & $(n=63)$ & $(n=176)$ & p \\
\hline Infection & $30.2 \%(19 / 63)$ & $19.3 \%(34 / 176)$ & $p=0.05$ \\
\hline Hypertension & $23.8 \%(15 / 63)$ & $22.7 \%(40 / 16)$ & $p=0.05$ \\
\hline Premature rupture of membranes & $22.2 \%(14 / 63)$ & $9.7 \%(17 / 176)$ & $\mathrm{p}<0.05$ \\
\hline \multicolumn{4}{|l|}{ Abortion } \\
\hline -artilicial & $7.9 \%(5 / 63)$ & $11.9 \%(21 / 176)$ & $p>0.05$ \\
\hline -spontaneous & $14.3 \%(9 / 63)$ & $12.5 \%(22 / 176)$ & $p=0.05$ \\
\hline Multiple pregnancy & $22.2 \%(14 / 63)$ & $21 \%(37 / 176)$ & $p=0.05$ \\
\hline In vitro fertilization & $12.7 \%(8 / 63)$ & $10.2 \%(18 / 176)$ & $p \div 0.05$ \\
\hline
\end{tabular}




\begin{tabular}{|c|c|c|c|c|c|c|}
\hline & \multirow{2}{*}{\multicolumn{3}{|c|}{$\begin{array}{c}\text { Apgar scores in the first minute of } \\
\text { life }\end{array}$}} & \multirow{2}{*}{\multicolumn{3}{|c|}{$\begin{array}{c}\text { Apgar scores in the fifth minute of } \\
\text { life }\end{array}$}} \\
\hline & & & & & & \\
\hline & $1=4$ & $5=7$ & $8=10$ & 1.4 & 5.7 & $8-10$ \\
\hline $\begin{array}{l}\text { Without sepsis } \\
(n-176)\end{array}$ & $\begin{array}{l}28.9 \% \\
(51 / 176)\end{array}$ & $\begin{array}{l}46.5 \% \\
(82 / 176)\end{array}$ & $\begin{array}{l}23.2 \% \\
(41 / 176)\end{array}$ & $\begin{array}{l}13.1 \% \\
(23 / 176)\end{array}$ & $\begin{array}{l}35.8 \% \\
(63 / 176)\end{array}$ & $\begin{array}{l}50.0 \% \\
(88 / 176)\end{array}$ \\
\hline Sepsis $(n=63)$ & $\begin{array}{l}49.2 \% \\
(31 / 63)\end{array}$ & $\begin{array}{l}33.3 \% \\
(21 / 63)\end{array}$ & $\begin{array}{l}17.5 \% \\
(11 / 63)\end{array}$ & $\begin{array}{l}15.9 \% \\
(23 / 63)\end{array}$ & $\begin{array}{l}55.6 \% \\
(35 / 63)\end{array}$ & $\begin{array}{l}28.5 \% \\
(18 / 63)\end{array}$ \\
\hline p & 0.004 & 0.058 & 0.316 & 0.602 & 0.008 & 0.003 \\
\hline
\end{tabular}

Table 4. The most frequently isolated bacterial species in blood cultures of newborns with confirmed sepsis

\begin{tabular}{|c|c|c|c|}
\hline Isolated bacteria & $\begin{array}{l}\text { Early sepsis } \\
\qquad n=23\end{array}$ & $\begin{array}{l}\text { Late sepsis } \\
\qquad \mathbf{n}=\mathbf{2 8}\end{array}$ & p value \\
\hline Staphylococcus aureus & $2 / 23(8.7 \%)$ & $0 / 28(0 \%)$ & $\mathrm{p} \gg 0.05$ \\
\hline Enterobacter spp. & $2 / 23(8.7 \%)$ & $2 / 28(7.1 \%)$ & $\mathrm{p} \gg 0.05$ \\
\hline $\begin{array}{l}\text { Staphylococcus spp. } \\
\text { (Coagulase negative) }\end{array}$ & $2 / 23(8.7 \%)$ & $2 / 28(7.1 \%)$ & $p \gg 0.05$ \\
\hline Klebsiella pneumoniae & $4 / 23(17.4 \%)$ & $1 / 28(3.6 \%)$ & $\mathrm{p} \gg 0.05$ \\
\hline Acinetobacter spp. & $9 / 23(39.1 \%)$ & $19 / 28(67.9 \%)$ & $\mathrm{p}<0.05$ \\
\hline Klebsiella pneumoniae & $1 / 23(4.3 \%)$ & $0 / 28(0 \%)$ & $\mathrm{p}>0.05$ \\
\hline Candida spp. & $1 / 23(4.3 \%)$ & $0 / 28(0 \%)$ & $p>0.05$ \\
\hline Citrobacter spp. & $0 / 23(0 \%)$ & $1 / 28(3.6 \%)$ & $p>0.05$ \\
\hline Escherichia coli & $2 / 23(8.7 \%)$ & $2 / 28(7.1 \%)$ & $\mathrm{p}>0.05$ \\
\hline $\begin{array}{l}\text { Streptococcus } \\
\text { agalactiae }\end{array}$ & $0 / 23(0 \%)$ & $1 / 28(3.6 \%)$ & $p>0.05$ \\
\hline
\end{tabular}

\begin{tabular}{|c|c|c|c|}
\hline \multirow[b]{2}{*}{ Bacterial species } & \multicolumn{2}{|c|}{ Blood culture } & \multirow{2}{*}{ p } \\
\hline & Group $1(n=22)$ & Group 2 ( $\mathrm{n}=8)$ & \\
\hline Staphylococcus aureus & $54.5 \%(12 / 22)$ & $.0 \%(0 / 8)$ & 0.006 \\
\hline Streptococcus agalactiae & $9.1 \%(2 / 22)$ & $12.5 \%(1 / 8)$ & 0.782 \\
\hline Enterobacter spp. & $9.1 \%(2 / 22)$ & $.0 \%(0 / 8)$ & 0.377 \\
\hline Candida albicans & $4.5 \%(1 / 22)$ & $.0 \%(0 / 8)$ & 0.540 \\
\hline Microcaccus spp. & $4.5 \%(1 / 22)$ & $.0 \%(0 / 8)$ & 0.540 \\
\hline \multicolumn{4}{|l|}{ Coagulase negative } \\
\hline Staphylococcus spp. & $13.6 \%(3 / 22)$ & $62.5 \%(5 / 8)$ & 0.007 \\
\hline Klebsiella pneumoniae & $.0 \%(0 / 22)$ & $12.5 \%(1 / 8)$ & 0.092 \\
\hline Escherichia coli & $.0 \%(0 / 22)$ & $12.5 \%(1 / 8)$ & 0.092 \\
\hline
\end{tabular}

\section{Discussion}

Neonatal sepsis most commonly occurs in prematurely born babies who underwent aggressive therapeutic mea ures (placement of central venous and arterial catheters, endotracheal intubation, pleural and/or abdominal drainage, etc.) in order to maintain vital functions. Another group of infants with a high risk are those born from pregnancies complicated with mother-related infections (maternal febrile conditions, mother urinary tract infection, premature rupture of membranes, especially more than $18 \mathrm{~h}$ before delivery, multiple pregnancies). In addition, risk factors for neonatal sepsis are low gestational age, low birth weight, long hospitalization in the intensive care unit, parenteral nutrition ${ }^{1,3,9}$. Correct diagnosis of sepsis is based on a combination of clinical and laboratory findings. Blood cultures findings (positive or negative) should be taken with a reserve due to low bacterial load, presence of biofilms or antibiotic treatment. Many studies have shown that newborns with sepsis often have negative blood culture findings. For the diagnosis of sepsis, in premature infants, blood culture should be taken, but the diagnosis should be set on the basis of other parameters, in particular regarding the values of procalcitonin and C-reactive protein ${ }^{10,11}$. Utomo and al. found that the most common risk factors for neonatal sepsis are meconial aspiration and premature birth. In their research, neonatal sepsis was more frequently diagnosed in babies born below the 30th week of gestation and lower birth weight ${ }^{12}$. Similar data were reported in the study of Stoll et al ${ }^{5}$. Also, in our study, neonatal sepsis occurred most frequently in newborns with lower gestation and lower birth weight. Schuchat et al. did not find any difference in the incidence of neonatal sepsis between males and females ${ }^{1}$. Those data are consistent with the results of our study. Shah et al. concluded that neonates who had low Apgar score (AS) $(<5)$ in the first minute of life have high risk for neonatal sepsis (13). Rudiger and al. have stressed out the fact that decreased AS $(<3$ in the first minute of life, and $<6$ in the fifth minute of life) increases the risk of morbidity and mortality, and alters the risk for infection ${ }^{14}$. In our study, AS 1-4 in the first minute of life, and AS 5-7 in the fifth minute of life, were recognized as risk factors for sepsis. Zhoughua et al. have concluded that intrauterine growth restriction may have no impact on the extrauterine life or the occurrence of sepsis ${ }^{15}$. Our 
study also did not find a relationship between intrauterine growth restriction and incidence of neonatal sepsis. On the other hand, Damodaram et al. have found that sepsis more is frequent in infants with intrauterine growth restriction ${ }^{16}$. In a study conducted by Khassawneh et al. neonatal sepsis was highly associated with pneumonia ${ }^{17}$. In our study, $82.5 \%$ of preterm infants with sepsis was mechanically ventilated, and even $38.1 \%$ of them were diagnosed with pneumonia, what was significantly more in comparation with group of infants without sepsis. Also, premature infants with sepsis more frequently had other associated diseases, such as meningitis and necrotizing enterocolitis. Hartojo et al. studied premature infants with umbilical venous catheters and came to the conclusion that the duration of catheterization has no impact on the occurrence of neonatal sepsis ${ }^{18}$. In our study, infants who had sepsis were more likely to have umbilical venous catheters. Ananthakrishnan et $\mathrm{al}^{1}$. in their research found Klebsiela pneumoniae as the most common cause of early neonatal sepsis and Khassawneh et al. showed that Staphylococcus aureus was the most common cause of late sepsis ${ }^{17}$. Dhumal et al. found that the most common cause of neonatal sepsis are Gram-negative bacteria - Klebsiella spp and Escherichia coli ${ }^{19}$. Pseudomonas aeruginosa and Staphylococcus aureus were the most frequently isolated pathogens in neonates with sepsis in a research of Orrett et $a{ }^{4}{ }^{4}$. It is known that Acinetobacter is an opportunistic bacteria and that it is not a common cause of infections in healthy individuals, but in prematurely born infants, who do not have mature immune system, Acinetobacter may cause fetal infections because it is highly resistant microorganism. Acinetobacter is an ubiquitous microorganism and also, is a part of physiological microflora of the gut, which explains why it may be found in intensive care units.

Beaufort et al. conducted research in the neonatal intensive unit and designated Acinetobacter spp as the most common cause of late sepsis in preterm infants ${ }^{6}$. Orrett et al. found that Acinetobacter spp was isolated only sporadically, in septic patients ${ }^{4}$. In our study, the most common cause of early and late sepsis was Acinetobacter spp. Other studies did not identify Acinetobacter as a cause of early neonatal sepsis. However, we assume that the reason why, in our study, Acinetobacter was the most frequently isolated causal agent of early neonatal sepsis is that during the study period NICU facilities were maximally filled up, what, at the same time, caused a relative shortage of nurses. It is important to realize that our NICU is placed a relatively small area that is shared with pediatric Intensive Care Unit (PICU), with joint staff for both units. Usually, PICU patients are chronically ill patients, with tracheotomy and dependent on long-term mechanical ventilation, whose respiratory tract is commonly colonized with numerous resistant bacteria, including Acinetobacter $\mathrm{spp}^{20}$. Blood cultures may be falsely positive due to contamination of the sample, or a false negative if newborns previously received antibiotics, or samples are taken in an inappropriate manner. Therefore, it is important to know the proper technique of taking blood for this type of analysis ${ }^{8}$. Developed countries invested very much in programs which intends to reduce the level of contaminations below $3 \%{ }^{8,21}$. Because contaminated blood cultures may induce high costs of treatment, this problem should be taken seriously. This is the reason why in many countries "sterile techniques" rather than "clean technique" were introduced as a standard for blood cultures sampling. ${ }^{8}$ The study of Souvenier et al. showed unsuited usage of antibiotics in $50 \%$ of patients with contaminated blood cultures ${ }^{22}$. Self et al, in their study, found that contamination rate was $4.3 \%$ at baseline and after the introduction of sterile technique of blood sampling (use of sterile sets) contamination rate was reduced to below $3 \%{ }^{8}$. Checklists were used to "round up" sterile technique of taking blood cultures and as a reminder to the staff members that during the intervention antiseptic conditions should be maintained and as a "manual" for a proper management with sets $^{8}$. Hall and colleagues found that after the introduction of sterile procedure for blood culture sampling, contamination rates of blood cultures decreased from $3.9 \%$ to $1.6 \%{ }^{21}$. In our study, the percentage of contaminated blood cultures was high $(16.4 \%)$ in the group 1 when the blood culture were taken using a "clean technique". The introduction of "sterile" procedures and check lists reduced rate of blood culture contamination to $7.6 \%$. Follow-up period in our study was short - only 6 months. Available data from the literature indicate that follow-up period should be longer, minimum 2 to 3 years $^{23}$. The persisting problem of contamination of blood cultures has implications for both patient care and laboratory services in developed and less developed countries. For patients, blood culture contamination results in the need for more cultures and additional diagnostic tests and unnecessary antimicrobial therapy. For the laboratories, comprehensive workup of contaminant isolates adds to the technologist workload and health care $\operatorname{costs}^{24}$. Recent survey conducted in the United States showed that false diagnoses based on contaminated blood cultures have been associated with more than $\$ 4,000$ in excess charges per-patient; more than 4 days of excess hospitalization; and increased resource utilization, including laboratory charges ${ }^{25}$. Blood culture contamination can be minimized through careful drawing of blood under scrupulous aseptic conditions and by the use of skin cleansing with locally available isopropyl alcohol and sterile sampling techniques. Application of these basic principles for blood cultures sampling in intensive care units in less developed countries may help reduce contamination rates and in so doing help characterize the clinical significance of microorganisms like $\mathrm{S}$. epidermidis that are more increasingly being documented among pediatric patients in medical centres across Africa and in pediatric and neonatal critical care units worldwide ${ }^{26-29}$.

\section{Conclusion}

Bates et al. found that Staphylococcus aureus is the most frequent contaminants ${ }^{30}$. In our study, blood cultures were usually contaminated with coagulase-negative Staphylococcus spp and Staphylococcus aureus. The main risk factors for sepsis were prelabor rupture of membranes, low gestational age, low birth weight, mechanical ventilation, umbilical venous catheter placement, and abdominal drainage. Education, training and awareness of employees, use of sterile sets for blood cultures sampling and checklists, permanent control of false positive blood cultures, as well as regular and routine monthly reports are key factors for success in reducing contamination rates.

\section{Conflict of interest statement}

The authors declare that they have no conflicts of interests.

\section{References}

1. Schuchat A, Zywicki SS, Dinsmoor MJ. Risk factors and opportunites for prevention of early-onset neonatal sepsis, a multicenter case-control study. Pediatrics 2000; 105(1), 21-26. 
2. Vasiljević B, Antonović O, Maglajlić-Đukić S, Gojnić M. Vrednosti C-reaktivnog proteina kod novorođenčadi sa sepsom. Srpski arhiv za celokupno lekarstvo. 2008; 136(5-6), 253-257.

3. Ohlin A. What is neonatal sepsis? Acta Pediatr. 2011; 100, 7-8.

4. Orrett FA, Shurland SM. Neonatal sepsis and mortality in a regional hospital in Trinidad: aetiology and risk factors. Ann Trop Paediatr. 2001; 21(1), 20-25.

5. Stoll BJ, Hansen N, Fanaroff AA, Wright LL, Carlo WA, Ehrenkranz RA. Late-onset sepsis in very low birth weight neonates. Neonatal research network pediatr. 2002; 110, 285-294.

6. Beaufort AJ, Bernards AT, Dijkshoorn, Vanboven L. Acinetobacter junii causes lifethreatening sepsis in preterm infants. Acta Paediatr. 1999; 88, 772-775.

7. Ananthakrishnan S, Gunasekaran D. Etiology and risk factors for early onest neonatal sepsis. Indian J Med Microbiol. 2009; 27(3), 279.

8. Self W, Speroff T, Grijalva GC, McNaughton C, Ashburn J, Liu D. Reducing blood culture contamination in the emergency department: an interrupted time series quality improvement study. AEM. 2013; 20 , 89-97.

9. Klinger G, Levy I, Sirota L, Boyko V, Reichman B, Lerner-Geva L. Epidemiology and risk factors for early onset sepsis among very-lowbirthweight infants. Am J Obstet Gynecol. 2009;201(1):38.e1-6.

10. Tuner D, Hammerman C, Rudensky B, Schlesinger Y, Schimmel $\mathrm{SM}$. The role of procalcitonin as a predictor of nosocominal sepsis in preterm infants. Acta Pediatr. 2006; 95, 1571-1576.

11. Arnon S, Litmanovitz I. Diagnostic tests in neonatal sepsis. Curr Opin Infect Dis. 2008;21(3):223-7.

12. Utomo M I. Risk factors of neonatal sepsis: a preliminary study in Dr. Soetomo hospital. IJTID. 2010; 1(1), 23-26.

13. Shah G, Budhathoki S, Das BK \& Mandal RN. Risk factors in early neonatal sepsis. Kathmandu Univ Med J. 2006; 4, 187-191.

14. Rudiger M, Kuster H, Herting E, Beger A, Muller C, Urlesberger B. Variations of Apgar score of very low birth weight infants in different neonatal intensive care units. Acta Pediatr. 2009; 98, 1433-1436.

15. Zhoughua E, Zhi KZ. Postnatal growth of very low birth weight infants during hospitalization. Chinese J of Ped. 2013;51(1), 4-11.

16. Damodaram M, Story L, Kulinskaya E, Rutherford M \& Kumar S. Early adverse perinatal complications in preterm growth-restricted fetuses. Austrl and New Zeal J of Obstet and Gynaecol. 2011; 51, 204 209.

17. Khassawneh M, Khader Y, Abuqtaish N. Clinical features of neonatal sepsis caused by resistant gram-negative bacteria. Pediatr International 2009; 51, 332-336.
18. Hartojo M, Utomo MI. Catheter duration and risk of sepsis in premature babies with umbilical vein catheters. IJTID. 2012; 3(1), 1014.

19. Dhumal P, Ujagare M, Gandham N, Nagdawane RP, Sardar M, Sharma M. Incidence and antimicrobial susceptibility pattern of neonatal septicaemia from tertiary care hospital of India. IJMCR. 2012; 7(3), 207-211.

20. Manchanda V, Sanchaita S, Singh NP. Multidrug Resistant Acinetobacter. J Glob Infect Dis. 2010; 2(3):291-304

21. Hall RT, Domenico HJ, Self WH, Hain PD. Reducing the blood culture contamination rate in a pediatric emergency department and subsequent cost savings. Am Acad of Pediatr. 2013;131: 292-297.

22. Souvenir D, Anderson DE, Palpant S, Mroch H, Askin S, Anderson J. Blood cultures positive for coagulasa-negative staphylococci: antisepsis, pseudobacteriemia and therapy of patients. J Clin Microbiol. 1998; 36(7), 1923-1926.

23. Hall, K. K., and J. A. Lyman. Updated review of blood culture contamination. Clin. Microbiol. Rev. 2006; 19:788-802

24. Weinstein M. P. Blood culture contamination: persisting problems and partial progress. J Clin Microbiol 2003; 41:2275-2278

25. Basurrah M. M, Madani T. A. Handwashing and gloving practice among health care workers in medical and surgical wards in a tertiary care centre in Riyadh, Saudi Arabia Scand J Infect Dis. 2006; 38:620624.

26. Ako-Nai A. K, Adejuyigbe E. A, Ajayi F. M, Onipede A.O. The bacteriology of neonatal septicaemia in Ile-Ife, Nigeria J Trop Pediatr 1999; 45:146-151.

27. Boukadida, J, Ben Abdallah H, Boukadida N. Profile and sensitivity to antibiotics of 115 staphylococcal strains implicated in septicemia in a Tunisian general hospital. Bull Soc Pathol Exot 2003; 96:283-285.

28. Osinupebi O. A, Olajubu F.A. Bacteraemia-a Sagamu perception. Afr J Med Med Sci 2003; 97:1714-1718.

29. Archibald L.K, Pallangyo K, Kazembe P, Reller L.B. Blood culture contamination in Tanzania, Malawi, and the United States: a microbiological tale of three cities. J Clin Microbiolog 2006; 44(12): 4425-4429

30. Bates DW, Goldman L, Lee TH. Contaminant blood cultures and resource utilization: the true consequences of false-positive results. JAMA 1991;265:365-369. 